\title{
A spectroscopic study of RCB stars in the Galaxy and the LMC
}

\author{
Karen Pollard ${ }^{1}$, P.L. Cottrell ${ }^{1}$, W.A. Lawson ${ }^{2}$ \\ ${ }^{1}$ Mount John University Observatory, Department of Physics and Astronomy, \\ University of Canterbury, Christchurch, New Zealand \\ ${ }^{2}$ Department of Physics, University College ADFA, University of \\ New South Wales, Canberra ACT 2600, Australia
}

\begin{abstract}
High resolution échelle spectra have been obtained of two Large Magellanic Cloud (LMC) and one galactic R Coronae Borealis (RCB) star with the AngloAustralian Telescope. An analysis of these data using $\mathrm{He}-$ and $\mathrm{C}$-rich models and the model atmosphere code WIDTH6 of Kurucz indicates that the galactic RCB star SU Tau and the two LMC stars, W Men and HV12842, have similar atmospheric parameters to the warmer galactic RCB and hydrogen-deficient Carbon ( $\mathrm{HdC}$ ) stars e.g., $\mathrm{R} \mathrm{CrB}$ and XX Cam. Specifically, the new stars have $\mathrm{T}_{\text {eff }} \sim 7000 \mathrm{~K}, \log g=0.5-1.0$, microturbulent velocities between 6 and 8 $\mathrm{km} . \mathrm{s}^{-1}$ and $\mathrm{C} / \mathrm{He}$ ratios from 0.004 to 0.006 .

An abundance analysis has been performed on these stars for a wide range of species. Special emphasis has been placed on particular elements $\mathrm{H}, \mathrm{He}, \mathrm{Li}$, $\mathrm{C}, \mathrm{N}, \mathrm{O}, \& \mathrm{Fe}$ (see figure 1), which are key indicators of the extent and relative importance of nuclear processes such as the CNO cycle and triple-alpha process, and also of the physical processes such as convective mixing and mass loss. Specific values for the abundances of $[\mathrm{H} / \mathrm{He}],[\mathrm{Li} / \mathrm{Fe}]$ and $[\mathrm{Fe} /$ total $]$ (expressed relative to the total abundance of all species) in SU Tau and HV12842 are -5.4 $\&-6.4,+2.5 \&+2.4,-0.4 \&-0.8$.
\end{abstract}

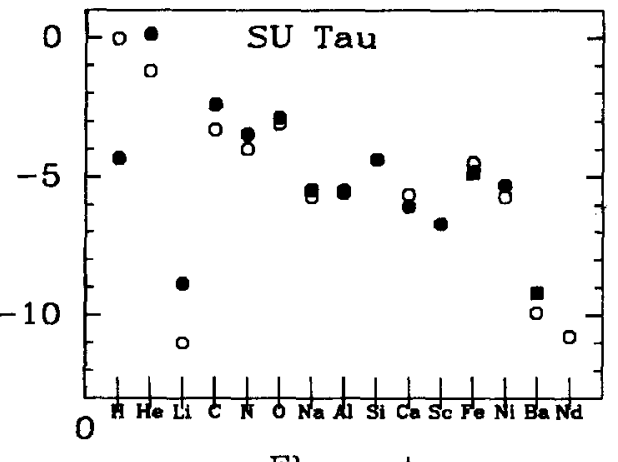

\section{Element}

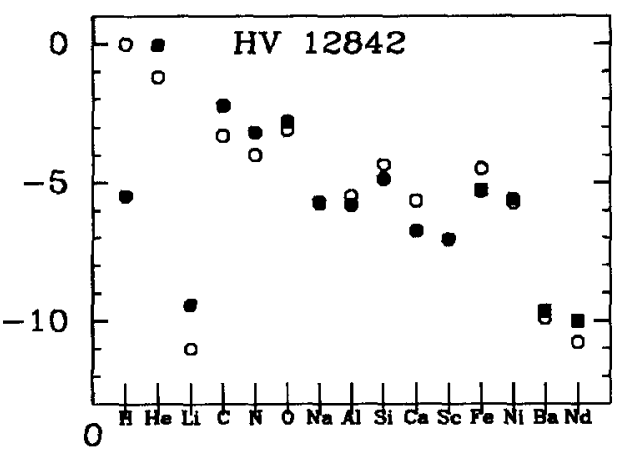

Element

Figure 1 Elemental abundances for (a) SU Tau and (b) HV12842, compared with those for the Sun. The RCB stars are shown with filled symbols and the Sun with open symbols. This figure shows the same general trend of abundances for the two RCB stars, with the large enhancement of $\mathrm{Li}$ and $\mathrm{N}$ (and of course He and $\mathrm{C}$ ). Hydrogen is depleted by more than $10^{4}$. Iron is depleted in the LMC object by a factor of $\sim 5$. 